\title{
Electric Fields on Oxidized Silicon Surfaces: Static Polarization of PbSe Nanocrystals ${ }^{\dagger}$
}

\author{
Chaya H. Ben-Porat, Oksana Cherniavskaya, and Louis Brus* \\ Chemistry Department, Columbia University, New York, New York 10027
}

\author{
Kyung-Sang Cho \\ IBM Research Division, T. J. Watson Research Center, Yorktown Heights, New York 10598
}

Received: November 10, 2003; In Final Form: February 26, 2004

\begin{abstract}
By use of electrostatic force microscopy, we measure the charge and polarizability of 12-nm PbSe nanocrystals on $\mathrm{n}$ - and p-type silicon with a 2-nm thermal oxide layer. Individual nanocrystals show a dielectric constant of $>100$. In ambient light, the nanocrystals generate static electric fields of magnitudes too weak to be caused by a full elementary charge. These nanocrystals are statically polarized by surface electric fields generated by fixed charges in the oxide substrate. We model this effect quantitatively and assign charge locations in the oxide. Upon 442-nm photoexcitation, we observe some of the nanocrystals $(\sim 35 \%)$ photoionize and slowly relax overnight back to their initial states. Just above a charge in the oxide, the surface electric field can approach $10^{8} \mathrm{~V} / \mathrm{cm}$.
\end{abstract}

\section{Introduction}

What type of electrostatic environment exists for molecules and nanocrystals deposited on thin (few nanometers) oxide layers on a Si wafer? How big is the induced molecular dipole due to the surface electric field? Buried silicon-silicon dioxide interfaces are typically partially charged due to carriers trapped on dangling bond interface states. This charging creates a strong dipole field (i.e., a band-bending field) at the $\mathrm{Si}-\mathrm{SiO}_{2}$ interface. Yet in the limit that the interface charge is modeled as a continuous sheet of charge and the oxide is a homogeneous classical dielectric, this electric field is completely screened at the oxide-vacuum interface a few nanometers away. However, the electric field on real oxide surfaces is not completely screened due to (a) the discrete nature of the interface charge, (b) the possible presence of additional discrete static charges in the oxide, and (c) the fundamental fact that the oxide is amorphous with varying local electrical properties. ${ }^{1-6}$ We now study these electric fields, and their effect on adsorbed $\mathrm{PbSe}$ nanocrystals, using a direct imaging method: electrostatic force microscopy (EFM). We also photoexcite the samples in order to vary the surface charge distribution.

Semiconductor nanocrystals (in the IV-VI group) have potential applications in the mid-infrared as optoelectronic emitters, sensors, and detectors. ${ }^{7-11}$ An interesting feature of IV-VI semiconductor materials such as $\mathrm{PbSe}$, in contrast to most II-VI and III-V materials, is the much larger size of the exciton Bohr radius (46 nm). ${ }^{12-14}$ Hence strong quantum confinement effects in larger nanocrystals can be observed. Bulk $\mathrm{PbSe}$ has a cubic (rock salt) crystal structure, a narrow direct band gap $(0.21 \mathrm{eV}$ at $300 \mathrm{~K})$, high dielectric constant $(\epsilon=250)$, and high carrier mobility. ${ }^{14}$ Until recently, ${ }^{15-17}$ few studies have been done to investigate the properties of PbSe nanocrystals

\footnotetext{
† Part of the special issue "Richard Bersohn Memorial Issue".

* To whom correspondence may be addressed. E-mail: brus@ chem.columbia.edu.

$\doteqdot$ Also associated with ARMI, University of New Orleans, LA. Present address: InnovaLight Inc. 12024 Vista Parke Drive, Austin, TX 78726 4050 .
}

compared to III-V materials because of the lack of synthetic methods to yield high-quality nanocrystals. Previous studies ${ }^{18,19}$ in our lab have shown blinking and photoinduced charging properties of 4-5-nm individual CdSe nanocrystals on thin oxides. Here we report a study of the electrostatic charge distribution and photoionization properties of single $\mathrm{PbSe}$ nanocrystals.

\section{Experimental Section}

EFM $^{20-27}$ simultaneously measures surface topography and the electrostatic force gradients above the surface. A conductive probe is electrically connected to the conducting substrate. Two passes are made for each line: the first records a regular tapping mode topography of the sample; the second pass is used to measure the shift of the resonance frequency of the tip when it is lifted, $z_{\text {lift }}$, above the substrate and dithered mechanically at its natural frequency while applying a voltage, $V_{\mathrm{DC}}+V_{\mathrm{AC}} \sin$ $(\omega t)$. With lock-in detection, we can isolate two oscillating components of the electric field gradient, one at the frequency of the applied voltage, $\omega$, and the other at twice that frequency, $2 \omega$. The $\omega$ signal is due to static electric fields from charges and/or multipoles in the sample, and the $2 \omega$ signal is due to the sample polarizability. To simplify the signal, we null out the contact potential difference $\varphi$ between substrate and probe by setting $V_{\mathrm{DC}}=-\varphi$. By use of analytical models previously developed and calibrated ${ }^{18,28}$ to correctly describe the tipsample capacitance, we can determine the charge and dielectric constant of individual nanocrystals.

$\mathrm{PbSe} /$ oleic acid capped nanocrystals (12-nm diameter, with $\sim 8 \%$ size distribution) are synthesized by organometallic methods. ${ }^{29}$ EFM experiments are performed in a glovebox (MBraun Unilab, Simatic OP7), under nitrogen atmosphere at room temperature $\left(P\left(\mathrm{O}_{2}\right)<2 \mathrm{ppm}, P\left(\mathrm{H}_{2} \mathrm{O}\right)<1 \mathrm{ppm}\right)$. The nanocrystals are spin coated onto degenerately doped p-type (B-doped, 0.005-0.01 $\Omega \mathrm{cm}$ ) and n-type (Sb-doped, 0.008$0.03 \Omega \mathrm{cm}$ ) silicon substrates with a $2-\mathrm{nm}$ silicon dioxide layer (obtained from IBM Research, Yorktown Heights, NY) made by high-temperature oxidation. The substrates are cleaned with 
(a)

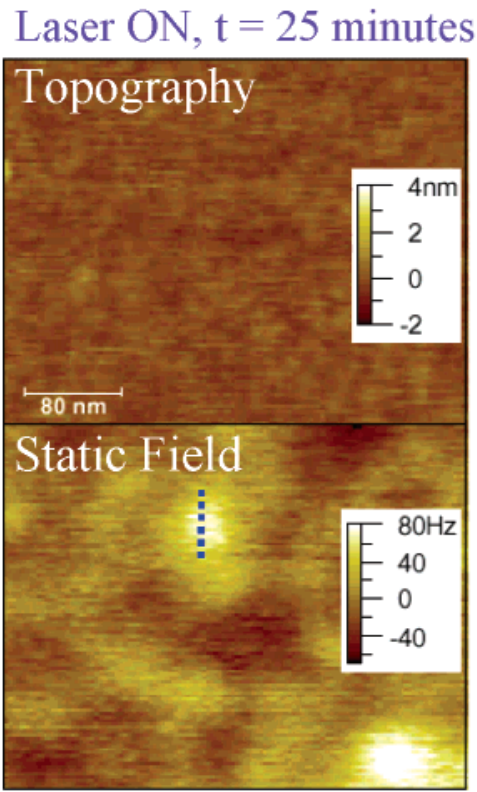

(b)

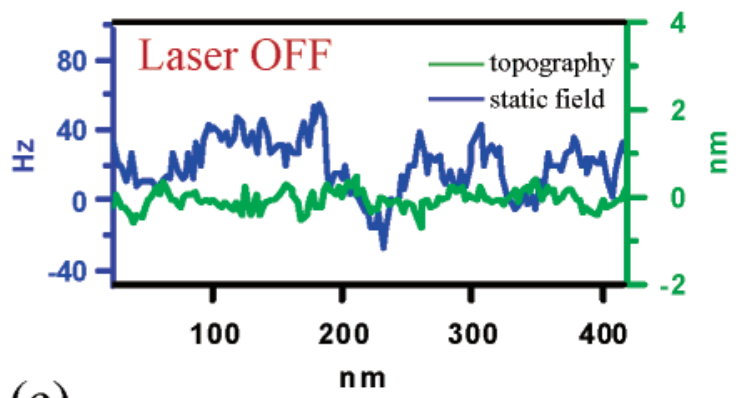

(c)

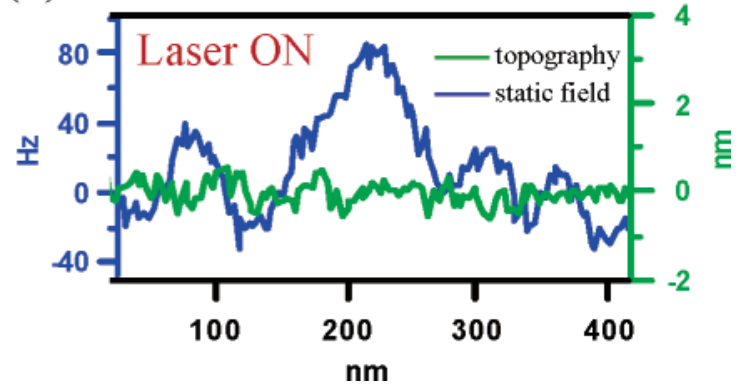

Figure 1. Bare substrate (n-type Si). (a) Topography and static field images of bare substrate photoexcited with 442-nm laser light. (b) Topography and static field line traces for the area marked in (a), laser OFF; (c) laser ON

ethanol and hexane prior to spin coating. Before deposition onto the substrates, the nanocrystal solution (hexane solvent) is stored in the dark, in the glovebox under the conditions mentioned above. Exposure of the samples to atmosphere is minimized to $\sim 10$ min during sample preparation. The EFM apparatus and methods have been previously described. ${ }^{18}$ The experiments are performed twice, once in the "dark" (i.e., exposed only to ambient light) and then repeated under the same conditions with photoexcitation of the sample (grazing angle) using a 442-nm laser (HeCd laser, Laconix 200 series), at $\sim 200 \mathrm{~mW} / \mathrm{cm}^{2}$. It takes $\sim 11$ min to collect each frame. We use Pt-Ir-coated EFM tips (Nanosensors EFM-20, Molecular Imaging, Phoenix, AZ) with resonance frequencies around $65 \mathrm{kHz}$ and spring constants measured to be around $1.35 \mathrm{~N} / \mathrm{m}$. All experiments are done using 2 calibrated probes with tip radii determined to be 25.4 and $25.7 \mathrm{~nm}$. Mathematical modeling was done using Mathematica 4.0 (Wolfram Research, Champaign, IL).

\section{Results}

1. Bare Oxide Substrate. A typical topography and static electric field image of photoexcited bare oxide (n-type $\mathrm{Si}$ ) is shown in Figure 1a, for a lift height of $11 \mathrm{~nm}$. Image contrast in static field image arises from static electric field gradients at the height of the tip and may be due to charges, dipoles, or higher moments within the sample. The photoexcited image shown in Figure 1a corresponds to $\sim 25 \mathrm{~min}$ of exposure to 442-nm light. The topography and static field lines traces in parts $\mathrm{b}$ and $\mathrm{c}$ of Figure 1 are taken before and during photoexcitation, respectively. The oxide is flat with a roughness of just several angstroms. Both traces (parts b and c of Figure 1) correspond to the same area on the substrate, and the higher static field signal (blue trace, Figure 1c) clearly shows a photoinduced electric field source. Constant height and polarizability (not shown) are observed for all these images. Similar results are found on p-type Si.

2. PbSe Nanocrystals. In these experiments, 57 individual $\mathrm{PbSe}$ nanocrystals are studied on n-and p-type $\mathrm{Si}$ substrates. Figure 2 shows typical images of $\mathrm{PbSe}$ on p-type $\mathrm{Si}$. The three

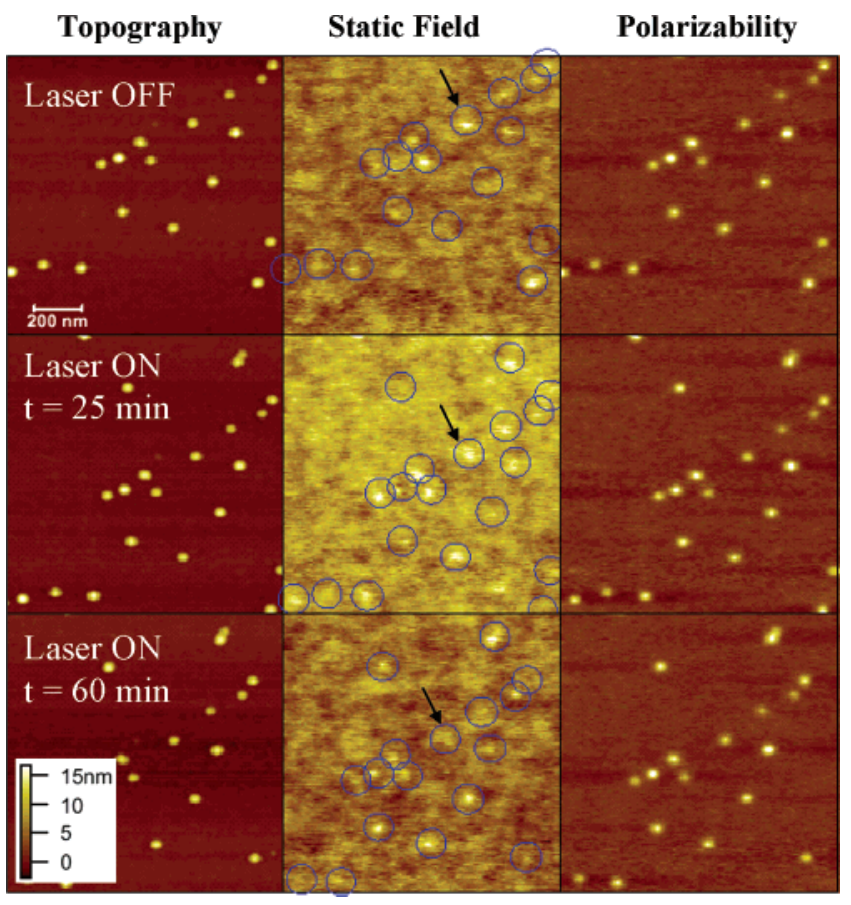

Figure 2. Topography, static field $\Delta v(2 \omega)$, and $\mathrm{AC}$ polarizability images of PbSe nanoparticles on p-type $\mathrm{Si}$ exposed only to room light (top) and 442-nm light (middle and bottom, at different times).

images from left to right correspond to (a) surface topography, (b) static field, and (c) polarizability. At a lift height of $20 \mathrm{~nm}$, $75 \%$ of the nanocrystals appear bright $(\Delta v(\omega)>40 \mathrm{~Hz})$ in the static field image under ambient light. The sign of the signal corresponds to a positive charge or to a dipole with the positive end up. About 25 min (1 h) after irradiation with 442-nm light, the middle (bottom) image appears qualitatively similar to the nonphotoexcited (top) image. However, the magnitudes of the static field signals are significantly higher. At $z=25.9 \mathrm{~nm}$ (we use $z$ to refer to the probe-substrate separation), the average observed signal for $\sim 15$ nanocrystals over 42 different measurements was $(0.29 \pm 0.12) \times 10^{-3} \mathrm{~N} / \mathrm{m}$ in room light and $(1.2 \pm$ 
(a)

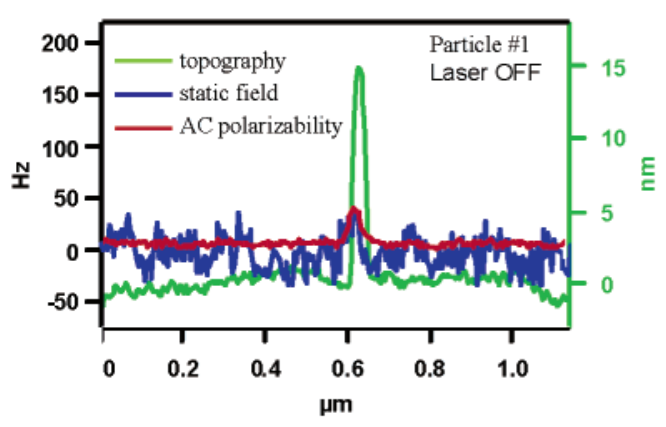

(c)

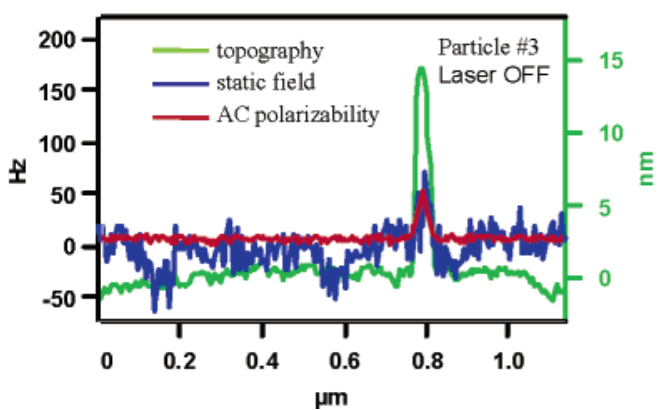

(b)

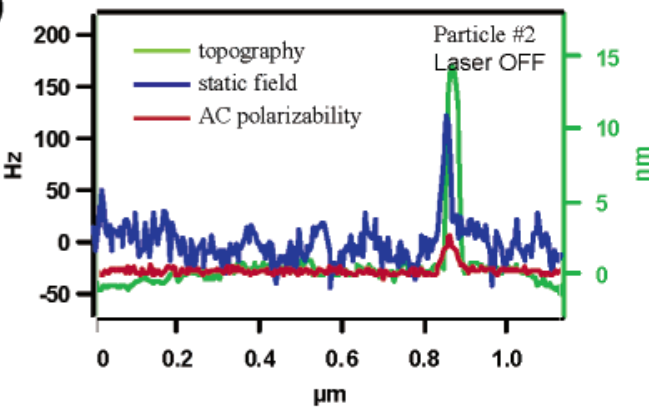

(d)

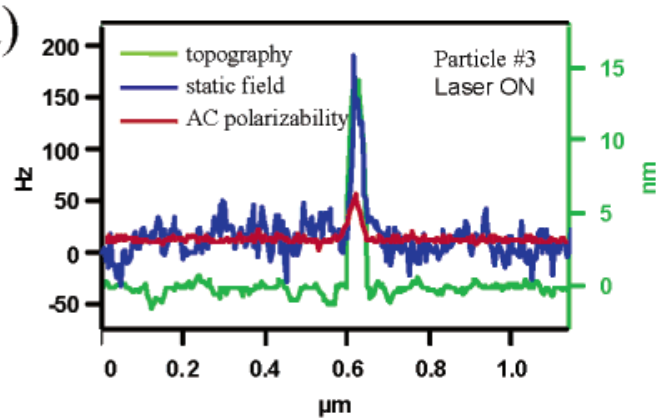

Figure 3. Experimental line scans of topography, static field, and AC polarizability for 3 individual nanocrystals on p-type Si. (a) Particle 1 shows no charge, (b) particle 2 a positive charge, (c) and (d) particle 3 shows an increase in the static field upon photoexcitation.

(a)
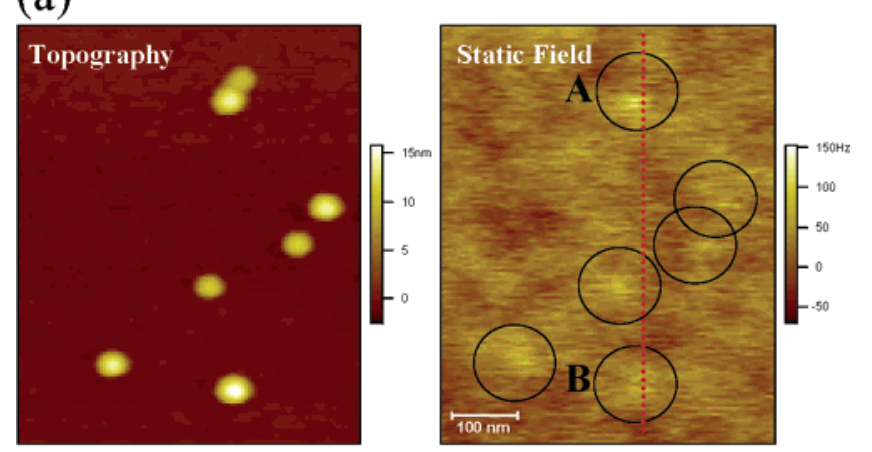

(b)

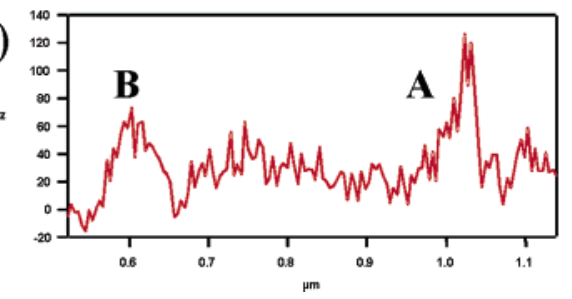

Figure 4. (a) Topography and static field image of PbSe nanocrystals on p-type Si exposed to 442-nm light. (b) Vertical line trace in the area of particles A and B. Blinking, or a change in positive signal on a time scale of $\sim 1 \mathrm{~s}$, is shown.

0.6) $\times 10^{-3} \mathrm{~N} / \mathrm{m}$ when photoexcited. The static field signal strength of individual nanocrystals showed large variations from one frame to the next (see for example the nanocrystal marked with an arrow in Figure 2). Parts $a-d$ of Figure 3 show experimental line scans for 3 typical nanocrystals: nanocrystal 1 in part a shows no static field signal (laser off), nanocrystal 2 in part b shows a weak signal (laser off), and nanocrystal 3 in parts $\mathrm{c}$ and $\mathrm{d}$ shows a weak signal (laser off) and a strong signal (laser on). We also see what appears to be a particle blinking from line-to-line as shown in Figure 4. The positive charge signal is going on and off with a frequency of $\sim 1 \mathrm{~s}$. The vertical line scan shown in Figure $4 b$ is a cross section of all the horizontal line scans that build up the image. Structure within the resolution-limited image of one nanocrystal reflects

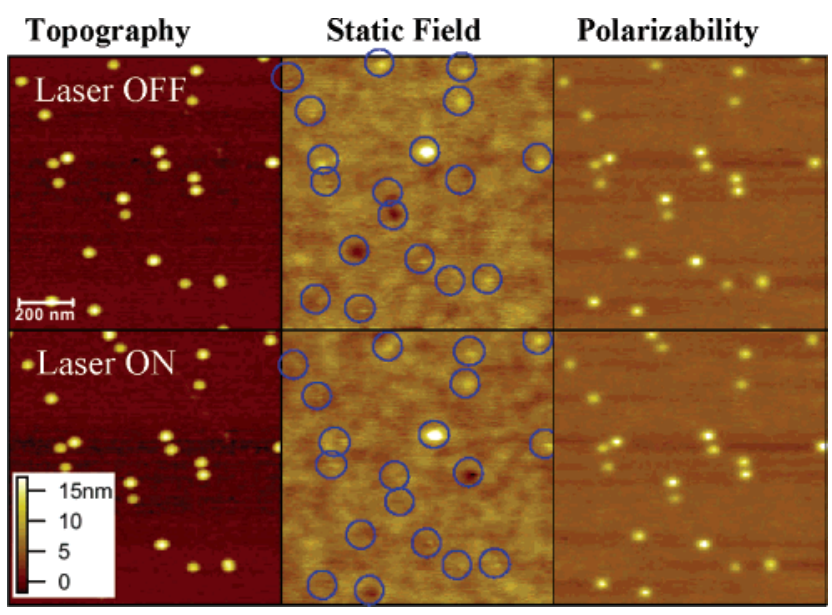

Figure 5. Topography, charge and polarizability images of $\mathrm{PbSe}$ nanocrystals on n-type Si exposed to room light (top) and 442-nm light (bottom). Note: dark areas correspond to negative charge.

changes in the static field strength on $\sim 1$-s intervals. This allows us to see the charge dynamics of an individual nanocrystal.

Similar static fields and nanocrystal polarizabilities are found on n-type $\mathrm{Si}$ as for p-type $\mathrm{Si}$. One difference we observe on n-type $\mathrm{Si}$ is the presence of some negative static fields from some of the nanocrystals (Figure 5). However, unlike the strong positive static fields that we observe during photoexcitation on both n- and p-type silicon, we do not find any negative fields with comparable magnitudes.

\section{Analysis}

A. Oxidized Silicon Substrate. There are dangling bond states at the $\mathrm{Si}-\mathrm{SiO}_{2}$ interface that can act as donors or acceptors and hence be positively or negatively charged. In addition, there can be buried point charges within the oxide layer or at the surface. We can calculate the field strength due to such a point charge buried in the oxide. We model the system in the following way (Figure 6): three regions of dielectric constants $\epsilon_{1}, \epsilon_{2}, \epsilon_{3}$ represent ambient (region I), oxide (region II), and 


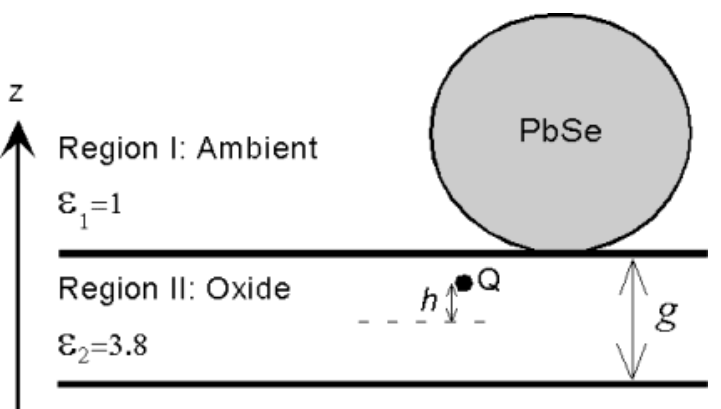

Region III: Doped Silicon

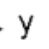

Figure 6. Three regions of varying dielectric constants, with point charge $Q$. Coordinates $z$ and $y$ are labeled.

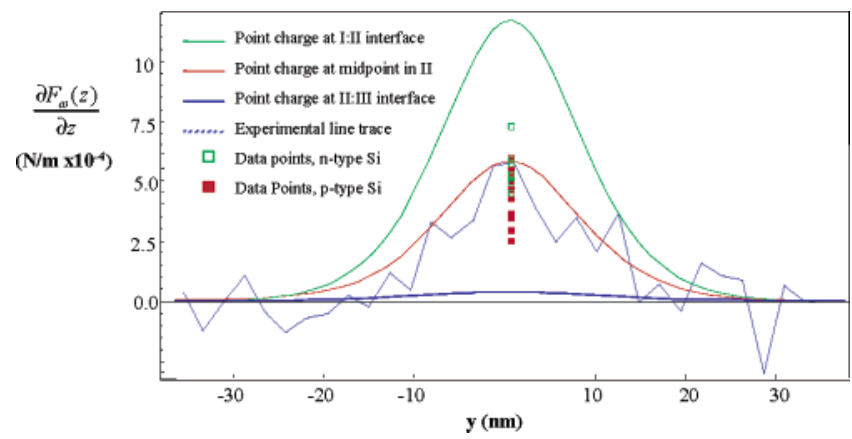

Figure 7. Plot of $\partial F_{\omega}(z) / \partial z$ vs $y$, at $z=17 \mathrm{~nm}$. Model plotted for three point-charge locations with experimental data points and a typical experimental line trace.

the doped (region III) silicon, respectively. A point charge $Q$ is in region II of thickness $g=2 \mathrm{~nm}$ at a distance $h$ from the midpoint. An infinite set of image charges must be used to represent the potential $V_{\mathrm{I}}(x, y, z)$ in region $\mathrm{I}^{30}$

$$
\begin{aligned}
V_{\mathrm{I}}(x, y, z) & =\frac{Q\left(1+k_{2}\right)}{4 \pi \epsilon_{0}}\left\{\frac{1}{\left(x^{2}+y^{2}+(z+h)^{2}\right)^{1 / 2}}+\right. \\
& \left.\sum_{i=0}^{\infty}\left(\frac{a_{i}^{*}}{\left(\frac{\left.x^{2}+y^{2}+\left(z+2 g\left(i+\frac{1}{2}\right)+h\right)^{2}\right)^{1 / 2}}{b_{i}^{*}}+\right.} \frac{\left(x^{2}+y^{2}+(z+2 g(i+1)-h)^{2}\right)^{1 / 2}}{z}\right)\right\}
\end{aligned}
$$

where $a_{i}^{*}=k_{3}^{i+1} k_{2}^{i}, b_{i}^{*}=k_{3}^{i+1} k_{2}^{i+1}, k_{2}=\left(\epsilon_{1}-\epsilon_{2}\right) /\left(\epsilon_{1}+\epsilon_{2}\right)$, and $k_{3}$ $=\left(\epsilon_{1}-\epsilon_{3}\right) /\left(\epsilon_{1}+\epsilon_{3}\right)$. The point $r=0$ corresponds to the midpoint of region II.

To test if our model yields field gradients in agreement with experiment, we plot in Figure 7 the experimental data points collected from bright spots in the bare substrate, superimposed onto a typical experimental line scan and the calculated signal due to a point charge in the oxide at three different $h$. The experimental data correspond to point charges lying near the middle of the oxide. The experimental line width in Figure 7 agrees with the theoretical line width $(\sim 40 \mathrm{~nm})$; in many cases, however, it is larger, implying more than one charge in the oxide.

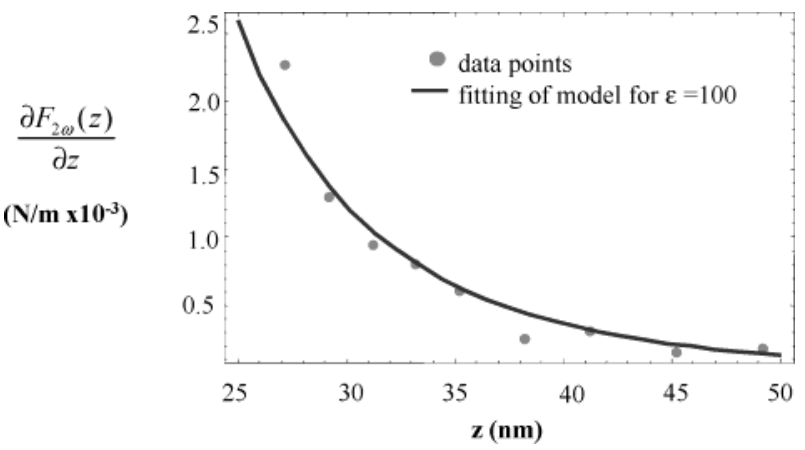

Figure 8. Plot of $\partial F_{2 \omega}(z) / \partial z$ vs $z$, fitting of the model (with $\epsilon=100$ ) to the experimental data for a single $\mathrm{PbSe}$ particle.

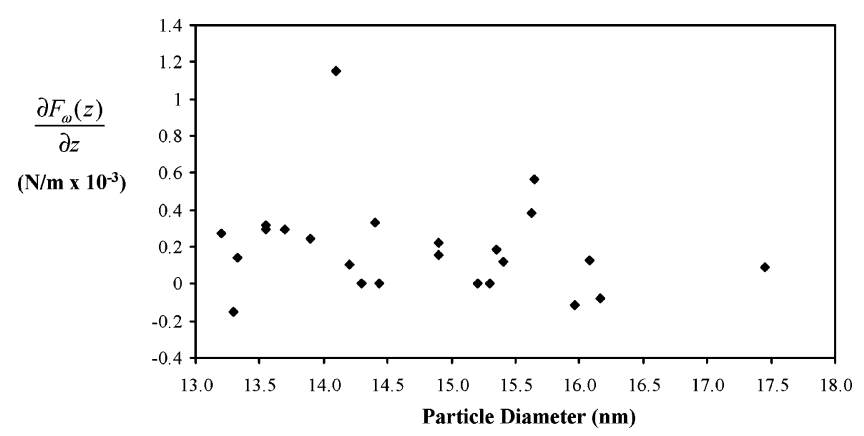

Figure 9. Plot of $\partial F_{\omega}(z) / \partial z$ vs particle diameter. The plotted data points represent 25 individual nanocrystals with a range of diameters. The probe-substrate separations, $z$, for these measurements are twice the diameter of the particle $( \pm 0.5 \mathrm{~nm})$.

The experimental data for bare substrate when photoexposed, such as the bright spots seen in Figure 1b, corresponds in our model to a point-charge location that is much closer to the $\mathrm{SiO}_{2}$-air interface when compared to the same substrate in the "dark". But increased signal strength may also be due to several unresolved charges in the oxide.

We can now address the question of what kind of electrostatic environment exists for molecules and nanocrystals on thin oxides. We calculate a field strength of $4.1 \times 10^{6} \mathrm{~V} / \mathrm{cm}$ at a probe-substrate separation of $z=3 \mathrm{~nm}$, when directly above a charge in the center of the oxide $(h=0)$. The field strength falls off very rapidly as we move away from the charge laterally; at $(y, z)=(3,3) \mathrm{nm}$ the field is $0.17 \times 10^{6} \mathrm{~V} / \mathrm{cm}$. At $z=0.5 \mathrm{~nm}$ above the substrate where an adsorbed molecule might be, the field is $1.0 \times 10^{8} \mathrm{~V} / \mathrm{cm}$ for $y=0$ and $1.5 \times 10^{6} \mathrm{~V} / \mathrm{cm}$ for $y=$ $3 \mathrm{~nm}$.

B. PbSe $\Delta v(2 \omega)$ AC Polarizability. From the $\Delta v(2 \omega)$ signal, we evaluate the field at the tip due to the AC polarization of a PbSe nanocrystal (measured at $800 \mathrm{~Hz}$ ). By use of the model described previously ${ }^{28}$ and the measured diameter for each nanocrystal, we calculate the dielectric constant of single PbSe particles. We fit the value of $\epsilon$ to the experimental data collected for a range of probe-substrate distances, $z$ (Figure 8). Fitting this for $\sim 50$ distinct nanocrystals yields a calculated dielectric constant of $\sim 100$ (at this signal-to-noise level, the model cannot discriminate with a high degree of accuracy between values $>100$ ), consistent with the experimental value for bulk $\mathrm{PbSe}$ of $250.0 .^{31}$ The polarizability behavior was uniform for the majority of the nanocrystals and did not depend on laser excitation or on the nature of the substrate (nor p-type $\mathrm{Si}$ ).

C. PbSe $\Delta v(\omega)$ Charge and Static Dipole. By use of our previously developed model, ${ }^{32}$ we find that the experimental static field observed for the majority of nanocrystals is too small to represent an ionized nanocrystal, regardless of the charge 
(a)

Particle \#4: $14.7 \mathrm{~nm}$ diameter

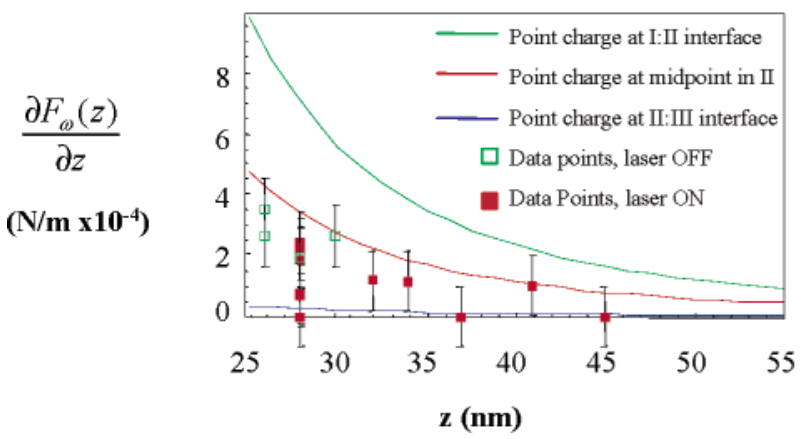

(b)

Particle \#5: $13.3 \mathrm{~nm}$ diameter

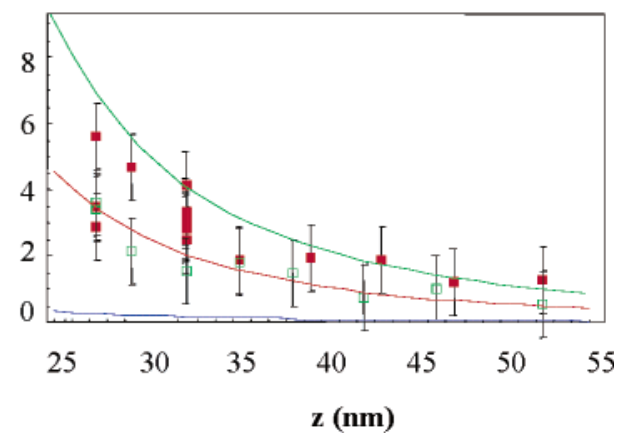

Figure 10. Point-charge model results plotted with experimental data, $\partial F_{\omega}(z) / \partial z$ vs $z$. (a) Particle 4, p-type Si; (b) particle 5, n-type Si.

location inside the nanocrystal. Also, since the measured dielectric constant in section B implies essentially metallic screening, the signal cannot be due to a separated electron hole pair in the nanocrystal. As shown in Figure 9, we find that the static signal is not correlated with the measured diameter, and thus is not due to a systematic structural dipole.

We postulate that the signal originates in nanocrystals that are polarized by the static charges in the 2-nm silicon oxide layer. Normally at the probe-substrate separations $z(\sim 25-50$ $\mathrm{nm}$ ) used for imaging of nanocrystals, the direct static field due to the oxide charges without surface $\mathrm{PbSe}$ nanocrystals leads to signals that are too small $\left(4.3 \times 10^{3} \mathrm{~V} / \mathrm{cm}\right.$ at $z=35 \mathrm{~nm}$ or $0.04 \times 10^{-3} \mathrm{~N} / \mathrm{m}$ ) to be detected by our apparatus. To test this idea, we add a term to (1) to account for the fields in region I due to a dielectric nanocrystal polarized by the point charge $Q$. The potential due to a nanocrystal being polarized by $V_{\mathrm{I}}$ is ${ }^{33}$

$$
\begin{aligned}
\xi(r)=\frac{1}{4 \pi \epsilon_{0}} \sum_{n=0}^{\infty} \frac{n(\epsilon-1)}{\epsilon n+n+1}\left\{\sum_{i=0}^{\infty} \frac{q_{i}^{*}(d / 2)^{2 n+1}}{r^{n+1} s_{i}^{n+1}}+\right. \\
\\
\left.\sum_{j=1}^{\infty} \frac{q_{j}^{*}(d / 2)^{2 n+1}}{r^{n+1} s_{j}^{n+1}}\right\} P_{n}(\cos \theta)
\end{aligned}
$$

where $d$ is the nanocrystal diameter, $q_{i}^{*}=Q\left(1+k_{2}\right) k_{3}^{i} k_{2}^{i}, q_{j}^{*}=$ $Q\left(1+k_{2}\right) k_{3}^{j} k_{2}^{j-1}, s_{i(j)}$ is the distance from the center of the nanocrystal to the $i$ th (jth) point charge, where $s_{i}=d / 2+g+$ $(4 i+1) h, s_{j}=d / 2-g+(4 j-1) h$, and $r=z-d / 2-g$ (the origin is set as the center of the nanocrystal). In this expression, $P_{n}$ is the $n$th Legendre polynomial, with $\theta$, the angle vector $r$ makes with the $z$ axis set to $0^{\circ}$, and $\epsilon$ is the dielectric constant of $\mathrm{PbSe}$, set to 250 (bulk value).

We calculate the force gradient on the tip due to both potentials $\left(V_{\mathrm{I}}+\xi\right)$ and plot it as a function of $z$ together with the experimental data in ambient light (Figure 10). The force gradient is plotted for three possible point-charge locations: slightly above the $\mathrm{Si}-\mathrm{SiO}_{2}$ interface (blue trace), at the $\mathrm{SiO}_{2}-$ air interface (green trace), and at the midpoint of $\mathrm{SiO}_{2}$ (red trace).

As expected, the strongest predicted signal (green trace) corresponds to the case where the point charge is located directly beneath the nanocrystal, while the weakest predicted signal $(\sim 0$, blue trace) corresponds to the point charge that is slightly above the silicon. For a typical nanocrystal (nanocrystal 4) on p-type silicon shown in Figure 10a, most of the experimental signals correspond to the range where the point charge is located in the lower half of the oxide, while for nanocrystal 5 shown on n-type silicon (Figure 10b), the data range is mostly corre- sponding to point charges in the top half of the oxide layer. The former case was more common for both substrates. These data agree with the range of point-charge locations observed on the bare substrates (in ambient light). The reason we see any of these point charges at the lift heights operated in this experiment is due to the contribution of the field of the polarized nanocrystal, which in effect makes it appear as if the charge is closer to the tip. The nanocrystals serve as antennas for the point charge defects in the substrate below, magnifying and sharpening their signals.

Upon photoexcitation, some of the nanocrystals lose one $(\sim 35 \%)$ and sometimes two $(\sim 2 \%)$ electrons. However, for many of the nanocrystals that did not photoionize, we again measured weak static fields as can be explained by charges in the oxide. However, photoexcited nanocrystal signals correspond to locations of the point charge that are much closer to the oxide-air interface than for nonphotoexcited nanocrystals. Alternatively, this could be due to more charge in the oxide, or positively charged nanocrystals with negative charge trapped in the oxide. Our bare substrate experimental results show that photoexcitation leads to higher signals (suggesting point-charge locations that are closer to the air-oxide interface, or higher charge concentration).

\section{Conclusion}

We have found that $\mathrm{PbSe}$ nanocrystals are highly polarizable and magnify surface electric fields from point charges in the oxide layer on $\mathrm{n}$ - and p-type silicon. In our case, the PbSe polarization screens the static field due to $Q$ out of the nanocrystal interior. In doing so, the nanocrystal develops almost macroscopic electrostatic moments whose fields are felt above the surface at the local probe tip. The EFM experiment also allows us to directly observe these static oxide charges on bare oxide surfaces. Upon 442-nm photoexcitation, the number of oxide charges and the net nanocrystal polarization increases. Full photoionization of $\mathrm{PbSe}$ nanocrystals was also observed $\sim 35 \%$ of the time, with overnight relaxation to the original neutral state.

Acknowledgment. This work has been supported by the Columbia University MRSEC under NSF DMR 0213574 and by New York State under the NYSTAR program. Oksana Cherniavskaya gratefully acknowledges Lucent Technologies for her graduate fellowship. Chaya Ben-Porat gratefully acknowledges primary financial support from the Chemical Sciences, Geosciences and Biosciences Division, Office of Basic Energy Sciences, U.S. D.O.E. (DE-FG02-01ER15264). 


\section{References and Notes}

(1) Shamir, N.; van Driel, H. M. J. Appl. Phys. 2000, 88, 909

(2) Ludeke, R.; Cartier, E. Applied Physics Lett. 2001, 78, 3998.

(3) Marsi, M.; Belkhou, R.; Grupp, C.; Panaccione, G.; Taleb-Ibrahimi, A.; Nahon, L.; Garzella, D.; Nutarelli, D.; Renault, E.; Roux, R.; Couprie, M. E.; Billardon, M. Phys. Rev. B 2000, 61, R5070.

(4) Shamir, N.; Mihaychuk, J. G.; van Driel, H. M. J. Appl. Phys. 2000 88,896

(5) Bloch, J.; Mihaychuk, J. G.; van Driel, H. M. Phys. Rev. Lett. 1996, 77,920 .

(6) Shamir, N.; Mihaychuk, J. G.; van Driel, H. M.; Kreuzer, H. J. Phys. Rev. Lett. 1999, 82, 359.

(7) Boberl, M.; Heiss, W.; Schwarzl, T.; Wiesauer, K.; Springholz, G. Applied Phys. Lett. 2003, 82, 4065.

(8) Olkhovets, A.; Hsu, R. C.; Lipovskii, A.; Wise, F. W. Phys. Rev. Lett. 1998, 81, 3539.

(9) Shen, W. Z.; Yang, H. F.; Jiang, L. F.; Wang, K.; Yu, G.; Wu, H. Z.; McCann, P. J. J. Appl. Phys. 2001, 91, 192.

(10) Klann, R.; Hofer, T.; Buhleier, R.; Elsaesser, T.; Lambrecht, A. Appl. Phys. Lett. 1992, 61, 2866.

(11) Du, H.; Chen, C. L.; Krishnan, R.; Krauss, T. D.; Harbold, J. M.; Wise, F. W.; Thomas, M. G.; Silcox, J. Nano Lett. 2002, 2, 1321

(12) Rogacheva, E. I.; Tavrina, T. V.; Nashchekina, O. N.; Grigorov, S. N.; Nasedkin, K. A.; Dresselhaus, M. S.; Cronin, S. B. Appl. Phys. Lett. 2002, 80, 2690

(13) Okuno, T.; Lipovskii, A. A.; Ogawa, T.; Amagai, I.; Masumoto, Y. J. Lumin. 2000, 87-89, 491.

(14) Wise, F. W. Acc. Chem. Res. 2000, 33, 773.

(15) Chen, M.; Xie, Y.; Lu, J.; Zhu, Y.; Qian, Y. J. Mater. Chem. 2001, 11,518

(16) Lifshitz, E.; Bashouti, M.; Kloper, V.; Kigel, A.; Eisen, M. S.; Berger, S. Nano Lett. 2003, 3, 857 .
(17) Zhu, J.; Liao, X.; Wang, J.; Chen, H. Y. Mater. Res. Bull. 2001, 36,1169 .

(18) Cherniavskaya, O.; Chen, L.; Islam, M. A.; Brus, L. Nano Lett. 2003, 3, 497.

(19) Krauss, T. D.; O’Brien, S.; Brus, L. E. J. Phys. Chem. B 2001, 105,1725

(20) Leveque, G.; Bonnet, J.; Tahraoui, A.; Girard, P. Mater. Sci. Eng. B 1998, B51, 197.

(21) Weaver, J. M. R.; Wickramasinghe, H. K. J. Vac. Sci. Technol., B 1991, 9, 1562

(22) Schonenberger, C.; Alvarado, S. F. Phys. Rev. Lett. 1990, 65, 3162.

(23) Muller, F.; Muller, A. D.; Hietschold, M.; Kammer, S. Meas. Sci. Technol. 1998, 9, 734 .

(24) Fujihira, M. Annu. Rev. Mater. Sci. 1999, 29, 353.

(25) Hao, H. W.; Baro, A. M.; Saenz, J. J. J. Vac. Sci. Technol., B 1991 $9,1323$.

(26) Martin, Y.; Williams, C. C.; Wickramasinghe, H. K. J. Appl. Phys. 1987, 61, 4723.

(27) Nonnenmacher, M.; Oboyle, M. P.; Wickramasinghe, H. K. Appl. Phys. Lett. 1991, 58, 2921.

(28) Cherniavskaya, O.; Chen, L.; Weng, V.; Yuditsky, L.; Brus, L. E. J. Phys. Chem. B 2003, 107, 1525.

(29) Murray, C. B.; Gaschler, S. S. W.; Doyle, H.; Betley, T. A.; Kagan, C. R. IBM J. Res. Dev. 2001, 45, 47.

(30) Weber, E. Electromagnetic Fields, Volume I-Mapping of Fields; John Wiley \& Sons: New York, 1950.

(31) Chen, C.-L. Elements of Optoelectronics and Fiber Optics; School of Electrical and Computer Engineering, Purdue University, Irwin, Chicago: Boston, 1996.

(32) Cherniavskaya, O.; Chen, L.; Brus, L. J. Phys. Chem. 2004, 108 , 4946.

(33) Böttcher, C. J. F. Theory of electric polarization, 2nd ed.; Elsevier Scientific Pub. Co.: Amsterdam, 1973; Vol. 1. 